\title{
Beyond conventional secondary prevention in coronary artery disease - what to choose in the era of CANTOS, COMPASS, FOURIER, ODYSSEY and PEGASUS-TIMI 54? A review on contemporary literature
}

\author{
Daniel Kalbacher, Christoph Waldeyer, Stefan Blankenberg, Dirk Westermann \\ Department of Interventional and General Cardiology, University Heart Center Hamburg, University Hospital Hamburg-Eppendorf, Hamburg, Germany \\ Contributions: (I) Conception and design: D Westermann, S Blankenberg, D Kalbacher; (II) Administrative support: C Waldeyer, D Kalbacher; (III) \\ Provision of study materials or patients: D Westermann, C Waldeyer; (IV) Collection and assembly of data: D Kalbacher, D Westermann; (V) Data \\ analysis and interpretation: D Westermann, D Kalbacher, C Waldeyer; (VI) Manuscript writing: All authors; (VII) Final approval of manuscript: All \\ authors. \\ Correspondence to: Dr. Dirk Westermann. University Heart Center Hamburg, Martinistr. 52, 20246 Hamburg, Germany. Email: d.westermann@uke.de.
}

\begin{abstract}
Patients with established cardiovascular (CV) disease remain at dramatic residual risk for subsequent events, despite growing evidence in secondary prevention and wider dissemination of intensive treatment. This review focuses on new options in secondary risk prevention as presented by these five major randomized controlled trials (RCT): PEGASUS-TIMI 54, COMPASS, FOURIER, ODYSSEY and CANTOS. Three main therapeutic targets are addressed: residual cholesterol, residual inflammatory and residual thrombotic risk. All of the trials reviewed included patients with stable CV disease on optimal medical treatment with a surprising similar mortality. As of now, evolocumab, alirocumab and ticagrelor are on the market, while rivaroxaban and canakinumab are not yet licensed for the treatment of secondary prevention in CV disease. Although life-style modifications and better utilization of established medical treatment options will remain first-line strategy, new medication is just about to enter the market. Secondary prevention in coronary artery disease (CAD) holds a strong potential to reduce subsequent CV events, even $\mathrm{CV}$ death. It seems that a combination of an aggressive lipid-lowering treatment in combination with antithrombotic therapy could improve prognosis significantly (at least for distinct subgroups). Against this background, individual efficacy, risk, and costs have to be considered when identifying patients for each new regime.
\end{abstract}

Keywords: Secondary prevention; cardiovascular disease; coronary artery disease (CAD)

Submitted Mar 27, 2018. Accepted for publication Jun 16, 2018.

doi: $10.21037 /$ atm.2018.08.03

View this article at: http://dx.doi.org/10.21037/atm.2018.08.03

\section{Introduction}

Patients with established cardiovascular (CV) disease are prone to suffer from subsequent events including stroke, myocardial infarction (MI) and death (1-3). Moreover, $\mathrm{CV}$ disease has an important impact on medical resource utilization and associated costs (4).

Therefore, primary prevention will be key to address this growing problem. Nevertheless, although relevant prognostic improvements can be achieved by modifying the $\mathrm{CV}$ risk profile $(5,6)$, secondary prevention needs to be optimized in order to reduce the global burden of the disease as well as the incidence of secondary events. This includes medical and non-medical interventions, both confirmed in randomized clinical trials resulting in strong evidence (7). 


\section{Page 2 of 8}

\section{Residual risk and the need for novel options in secondary prevention}

The onset of atherosclerosis is complex and multiple factors drive further progression of the disease (8). Increasing age, higher body mass index, male gender and impaired renal function as well as classical risk factors like hypertension, smoking, hyperlipidemia, diabetes and genetic predisposition are associated with an increased risk for subsequent events. Most of these risk factors can be altered and need to be treated aggressively in patients with established CV disease (9). Despite growing evidence and wider dissemination of intensive treatment in the setting of secondary prevention patients with established CVD remain at dramatic residual risk.

The magnitude and composition of the residual risk vary substantially between patients and are influenced by numerous factors with partly synergistic effects (Figure 1). A relevant portion is due to residual cholesterol risk, in particular since the vast majority of CVD-patients fail the recommended low-density lipoprotein (LDL) targets (10). During the past decades the inflammatory component of the residual risk with high sensitivity C-reactive protein (hsCRP) as the most prominent marker got into the focus of research (11-13). The inflammatory pathway became even more promising since an anti-inflammatory strategy could also lower residual cholesterol risk due to reduction of the atherogenicity of LDL (14). Correspondingly, addressing primarily the cholesterol component with intensive statin therapy was associated with significant reduction of inflammatory markers. Residual cholesterol as well as a residual inflammatory risk can both be effectively treated by statin therapy. Initial trials could demonstrate a reduction of mortality rates up to $20 \%$. Yet, high-dosed statin therapy is not frequently used in clinical practice $(15,16)$.

Dual anti-platelet therapy (DAPT) and anticoagulation can both influence the "residual thrombotic risk" (Figure 1). For DAPT, it has been already demonstrated that $\mathrm{CV}$ outcome can be improved by a prolonged regime, yet at the cost of increased bleeding rates (17).

In patients diagnosed with $\mathrm{CV}$ disease, risk optimization for subsequent events and CV death are warranted, especially in high-risk patients. To address current medical options for these patients, we review the most relevant randomized controlled trials (RCT) in the field of residual risk reduction and discuss their relevance for daily clinical practice.
Kalbacher et al. Beyond conventional secondary prevention in CAD

\section{PEGASUS-TIMI 54, a trial with prolonged DAPT}

In 2015, PEGASUS-TIMI 54, a double-blind, randomized, controlled, multi-center study including 21,162 patients with stable coronary artery disease (CAD), defined as history of MI ( 1 to 3 years earlier) were assigned to ticagrelor $90 \mathrm{mg}$ bid, ticagrelor $60 \mathrm{mg}$ bid or placebo in a 1:1:1-fashion (18). After a median follow-up of 33 months, the primary combined endpoint of MI, stroke or $\mathrm{CV}$ death [major adverse CV and cerebral events (MACCE)] was significantly reduced in both ticagrelor subgroups (HR for $60 \mathrm{mg} v s$. placebo: $0.84 ; 95 \% \mathrm{CI}: 0.74-0.95 ; \mathrm{P}=0.004$ and HR for $90 \mathrm{mg} v s$. placebo: 0.85 ; $95 \%$ CI: $0.75-0.96$; $\mathrm{P}=0.008$ ) at the cost of an increase in major bleedings. Mortality was not affected in PEGASUS-TIMI 54.

\section{COMPASS, adding low dose anticoagulation to ASA (acetylsalicylic acid)}

Recently, at the ESC congress 2017, results of COMPASS were presented and published simultaneously. A total of 27,395 patients with established CV disease were included in this RCT and randomized to ASA, Rivaroxaban $5 \mathrm{mg}$ bid or the combination of ASA and rivaroxaban $2.5 \mathrm{mg}$ bid in a 1:1:1-fashion (19). Interestingly, the study was terminated earlier due to significant superiority of the active study treatment. This led to a mean follow-up of only 23 months. The primary endpoint, defined by MACCE was significantly reduced in the ASA + rivaroxaban group (HR vs. ASA: 0.76; $95 \%$ CI: 0.66-0.86; $\mathrm{P}<0.0001)$, mainly driven by a significant reduction in strokes and $\mathrm{CV}$ death $(\mathrm{P}<0.0001, \mathrm{CV}$ death $\mathrm{P}=0.02$, MI: $\mathrm{P}=0.14$ ), whereas no significant difference was found for ASA vs. rivaroxaban $5 \mathrm{mg}$ bid $(\mathrm{P}=0.12)$. Significantly increased bleeding rates were found for both Rivaroxaban groups compared to ASA alone (HR for rivaroxaban + ASA vs. ASA: 1.70, 95\% CI: 1.40-2.05; $\mathrm{P}<0.0001)$, but without significant differences for both fatal or symptomatic critical organ bleeding including intracranial hemorrhage. The overall net clinical benefit (defined as MACE + fatal or symptomatic bleeding into critical organ) for ASA + rivaroxaban $2.5 \mathrm{mg}$ bid $v s$. ASA remained significant $(\mathrm{HR}=0.80 ; 95 \%$ CI: 0.70-0.91; $\mathrm{P}=0.0005)$. Importantly, sub studies identified this therapy to be especially beneficial in patients with PAD or underlying heart failure. 


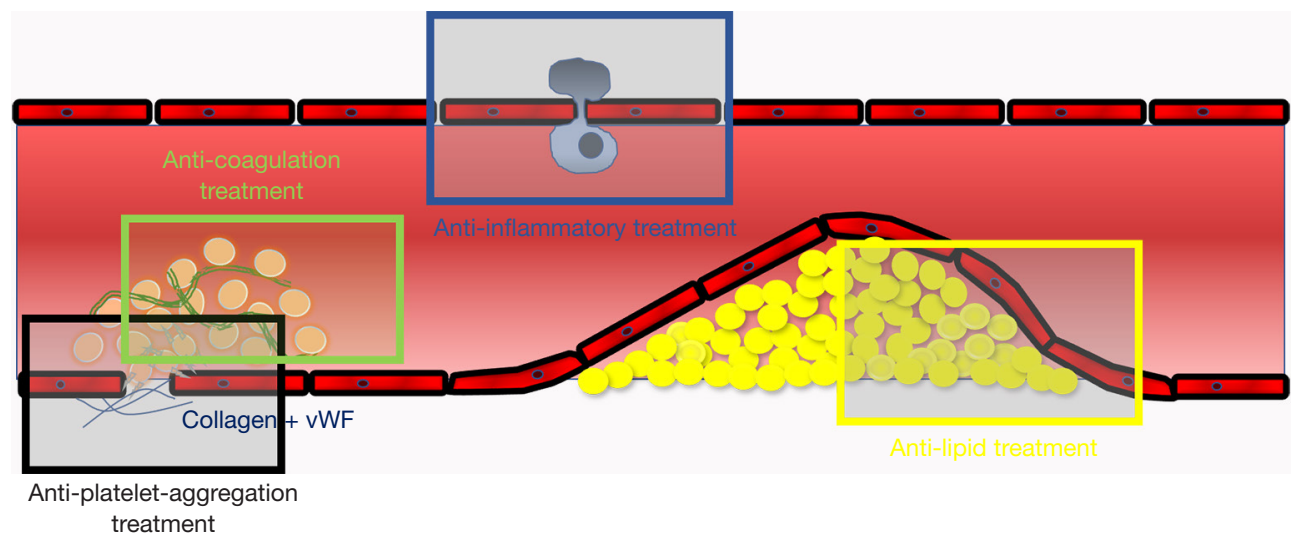

Figure 1 Residual risk in established cardiovascular disease. For all patients with known cardiovascular (CV) disease, the incidence of subsequent secondary events is high. There are 3 main mechanisms that contribute to the residual CV risk: first, cholesterol inclusions in the wall of blood vessels (highlighted by the yellow box). Second, inflammation processes can destabilize atherosclerotic plaques (highlighted by the blue box). Last but not least, injured endothelium or erupted atherosclerotic plaques activate the hemostasis bearing the risk of a complete plug of the vessel (primary hemostasis highlighted by both the black and secondary hemostasis highlighted by the green box). With PEGASUS-TIMI 54, COMPASS, FOURIER, ODYSSEY and CANTOS, 5 major RCT are focusing on secondary prevention in $\mathrm{CV}$ disease. Residual cholesterol risk and residual inflammatory risk can be addressed by statin therapy. Yet, for patients with excessive risk profiles, the human antibody evolocumab [a proprotein convertase subtilisin-kexin type 9 (PCSK9) inhibitor, FOURIER trial] offers a lipidlowering therapy beyond statins (yellow box). The CANTOS trial examining canakinumab (an interleukin-1 $\beta$ antibody) addressing residual inflammatory risk (blue box). It has already been demonstrated that statins can alter outcome by reducing inflammatory mechanisms. Yet, the concept of addressing interleukin-1 $\beta$ directly and thus reducing mortality and morbidity in CV disease has not been proven before CANTOS. The residual thrombotic risk can be addressed by inhibiting the function of thrombocytes (primary hemostasis, black box), which was examined in the PEGASUS-TIMI 54 trial using Ticagrelor, a P2Y12 receptor antagonist. The COMPASS trial focused on effects of rivaroxaban, a factor $\mathrm{Xa}$ antagonist inhibiting the coagulation cascade and thus preventing the formation of fibrin (green box).

\section{PCSK9 inbibition, maximal LDL reduction}

FOURIER: the first proprotein convertase subtilisin-kexin type 9 (PCSK9) inhibitor outcome-results were presented at the ACC congress in March 2017. The FOURIER trial randomised 27,564 patients with established $\mathrm{CV}$ disease (prior MI, prior stroke, PAD) and LDL levels $\geq 70 \mathrm{mg} / \mathrm{dL}$ (mean LDL at baseline $92 \mathrm{mg} / \mathrm{dL}$ ) despite moderate or high intensity statin therapy ( \pm ezetimibe) to receive the fully human monoclonal antibody evolocumab (140 mg every 2 weeks or $420 \mathrm{mg}$ every month, according to patient preference) vs. placebo as subcutaneous injection (20). The primary efficacy end point was defined as MACCE in addition to hospitalization for unstable angina (UA) or coronary revascularization and the key secondary end point was the defined as MACCE. The treatment arm had a significant reduction of LDL levels (59\% mean reduction, absolute reduction $56 \mathrm{mg} / \mathrm{dL}, 95 \% \mathrm{CI}$ : 55-57 mg/dL; $\mathrm{P}<0.00001)$ leading to less events for the primary endpoint (HR for evolocumab vs. placebo: 0.85; 95\% CI: 0.79-0.92;
$\mathrm{P}<0.0001)$ and key secondary endpoint (HR for evolocumab vs. placebo: 0.80; 95\% CI: 0.73-0.88; $\mathrm{P}<0.00001)$, but without significant differences for neither $\mathrm{CV}$ or overall mortality rates during a median follow-up of 2.2 years. A 12-month landmark analysis for MACCE identified a 25\% relative risk reduction for the second treatment year $(\mathrm{HR}$ for evolocumab vs. placebo, 12-month landmark: $0.75 ; 95 \%$ CI: $0.66-0.85 ; \mathrm{P}<0.00001)$. Notably, there were no relevant side-effects found under treatment with Evolocumab including screening for neurocognitive events and the development of neutralizing antibodies. In conclusion, the further lowering of LDL levels beyond defined thresholds in established $\mathrm{CV}$ disease was able to reduce $\mathrm{CV}$ events but did not affect CV or overall mortality rates.

ODYSSEY: alirocumab was tested in patients after recent ACS and presented at the ACC 2018. This trial investigated 18,924 patients with LDL levels $\geq 70 \mathrm{mg} / \mathrm{dL}$ (mean LDL at baseline $87 \mathrm{mg} / \mathrm{dL}$ ) despite high intensity statin therapy ( \pm ezetimibe) to receive the fully human monoclonal antibody Alirocumab (75 mg or up-titrated to $150 \mathrm{mg}$ every 
2 weeks) $v$ s. placebo as subcutaneous injection. With a similar endpoint (but without revascularization as an endpoint) it showed in a higher risk cohort with a longer follow up that Alirocumab reduced the primary endpoint by a total of $15 \%$. This was also true for all-cause death, which makes it the first trial reducing mortality with PCSK9 inhibition.

\section{CANTOS, blocking the inflammation pathway}

So far, no successful RCT was available proofing the concept of lowering residual inflammatory risk to improve outcome in CV diseases (11). The CANTOS study included 10,061 patients with prior MI on optimal medical treatment with hs-CRP levels $\geq 2 \mathrm{mg} / \mathrm{L}$ assigned to canakinumab, an interleukin-1 $\beta$ (IL-1 $\beta$ ) antibody functionally neutralizing the bioactivity of this cytokine (21). Canakinumab has a long half-life and provides significant reduction in IL$1 \beta$ and consequently CRP and IL-6 for up to 3 months. Patients were assigned to either 50, 150 or $300 \mathrm{mg}$ of canakinumab vs. placebo administered every 3 months by subcutaneous injection. The primary endpoint was MACCE and the secondary endpoint was MACCE and UA requiring coronary revascularization (MACCE+). The median followup time was 3.7 years.

A dose-dependent reduction in hs-CRP levels was noted (39\% reduction for the 150 and $300 \mathrm{mg}$ subgroups combined), whereas lipid levels were not affected. The best outcome was seen for patients treated with $150 \mathrm{mg}$ canakinumab (HR vs. placebo, primary endpoint: $0.85 ; 95 \%$ CI: $0.74-0.98 ; \mathrm{P}=0.021$; secondary endpoint: $0.83 ; 95 \%$ CI: $0.73-0.95 ; \mathrm{P}=0.005)$.

Additionally, there was a greater risk reduction for MACCE found in patients with falling hs-CRP levels $\geq$ median (of the whole cohort; 3-month follow-up) (HR vs. placebo: $0.73 ; 95 \% \mathrm{CI}$ : $0.63-0.83 ; \mathrm{P}=0.0001)$. Overall mortality rates as well as $\mathrm{CV}$ death were not affected. One important side-effect was the small absolute, but significant increment of fatal infections in the treatment arm due to immunomodulatory effects of canakinumab (incidence rate, 0.31 vs. 0.18 events per 100 person-years, $\mathrm{P}=0.02$ ). Notably, incidence of cancer was significantly reduced. While the absolute numbers were small, this is an interesting observation given the relative short duration of this study in view of primary cancer prevention.

\section{Discussion}

Given the current literature reviewed above, 4 distinct, new and additional medical options are available for the case presented here. Interestingly, all 5 trials target different biological pathways (2 with PCSK9 inhibition), therefore represent distinct interventions in the pathophysiology of atherosclerotic and atherothrombotic events. All of the trials included patients with stable CV disease on optimal medical treatment with a surprising similar mortality identifying similar patient characteristics. Yet, it is important to note that the included patients still vary. Whereas PEGASUSTIMI 54 and CANTOS only included patients with prior MI, both COMPASS and FOURIER also included patients without known CAD (but history of either prior stroke or PAD). ODYSSEY on the other hand included patients with previous ACS, making it the highest risk group of those trials. Obviously, this can affect outcomes, especially MI rates in the long run in the investigated populations.

Nevertheless, all trials investigated secondary prevention strategies for patients with enhanced risk and therefore are in contention of physicians' prescription behavior.

The effect size is comparable in all trials as well, especially when the primary endpoint is taken into account. While all trials have comparable primary endpoints, FOURIER included hospitalization UA and coronary revascularization, making interpretation of the results more difficult.

Mortality, including CV as well as all-cause mortality, is obviously the hardest endpoint did not reach statistical significance in neither of the studies presented (in COMPASS, there was a very clear trend HR $=0.82 ; 95 \%$ CI: 0.71-0.96; $\mathrm{P}=0.01$, yet the predefined threshold of $\mathrm{P}<0.0025$ was not met). ODYSSEY showed similarly reduced all-cause death while $\mathrm{CV}$ death failed to reach statistical significance but was numerically lowered as well.

The 12-month landmark analysis in the FOURIER trial gives reason to speculate that the biological pathways targeted in these trials are not all immediately affecting the current risk profile. The ODYSSEY trial, with markedly longer follow-up time, was therefore more likely to show an effect, especially when the higher risk profile is kept into mind. Whereas anticoagulatory effects targeted by PEGASUS-TIMI 54 and COMPASS are prone to reduce CV risk instantaneously, lipid lowering strategies are revealing their full potential at a later point in time. Large-scale data from numerous statin trials demonstrate a consistent time-dependent treatment effect with only slight risk-reduction in the first year and a stronger and stable risk reduction from the second year onwards (22). For the inflammatory pathway, it is unknown how long treatment 
has to be continued until risk reduction can be expected. This again underlines the point, that these trials are not easy no interpret, especially when it comes to measure effects.

Nevertheless, it is warranted to further reduce and control risk in stable CAD patients. Beyond guidelines and optimal medical treatment, it will also be the task for physicians to differentiate and evaluate the risk profile for the individual patient and on that basis to choose the optimal strategy. This means first and foremost medical treatment, as invasive strategies in stable CAD, are meant only for distinct patients (23).

\section{Additive effects?}

Since these additional treatment options are just about entering the market, there is no data on additive effects of these substances. The unmet need to address the residual risk multi-factorially became most obvious in the FOURIER trial. Despite achievement of unprecedented low levels of LDL near "LDL-eradication" the event rate in the study population was approximately $50 \%$ higher than postulated in the power calculation leading to a much shorter duration to accrue the prespecified number of events (20). Further analyses elucidating subgroups or markers of the relevant residual risk even at low and very low LDL levels in FOURIER or at reduced coagulation activity in COMPASS are eagerly awaited. Eventually, a combination of an aggressive lipid-lowering treatment strategy in combination with any antithrombotic concept (in a setting of low bleeding risk) might be a promising choice to significantly improve prognosis. Again, patients with high inflammatory markers may benefit most from antiinflammatory drugs. Yet, it has to be taken into account, that these new therapies are expensive and do burden the medical budgets.

\section{Cost issue?}

New medical treatment options are always costly and as these substances are just about to enter the market (as a treatment option for $\mathrm{CAD}$ ), prices are not yet available. For the sake of comparison, Table 1 contains available prices for the 5 substances (canakinumab is already available for rheumatic diseases, rivaroxaban for in the acute treatment of myocardial infarction and for atrial fibrillation, evolocumab, alirocumab and ticagrelor are on the market). The treatment costs vary significantly from about 1,200 EUR for rivaroxaban to more than 54,000 EUR for canakinumab per year (although that is expected to lower after FDA and EMA clearance). For medical health systems, it will always be challenging to distribute medical resources. Secondary prevention in CAD holds the potential to significantly reduce mortality and morbidity. Yet, since there are so many individuals affected of these diseases, cost issues will influence decision-making and prescription habits. Therefore, life-style modifications and better utilization of established medical treatment options will remain first-line strategy.

\section{Most bang for the buck}

Comparing those treatment strategies, it seems that the antithrombotic regimen with rivaroxaban is also the most cost-effective followed by PCSK9 inhibition with alirocumab.

For the lipid-lowering treatment, it has to be taken into account, that high-intensity statin therapy has shown to have the most significant impact in secondary CADprevention (16). At least at current costs PCSK9 inhibition via monoclonal antibodies will be an additional option mainly for those patients far above treatment targets or above treatment targets with recurrent events, as recommended by the ESC and EAS (24). Given that a post hoc analysis of CANTOS revealed better effects in patients which achieved the target hs-CRP, it may be an option to start treatment with one dose and decide to continue only in those with hs-CRP lower $2 \mathrm{mg} / \mathrm{L}$ (25). Yet, considering the current prices uptake in the general medical community is probably low for all biologicals.

\section{Perspective}

Effective secondary prevention in CAD is important to prevent morbidity and mortality. Additionally, modern societies are forced to consider cost-related factors as well. Although interventional treatment of CAD is the keystone in acute coronary syndromes, its role and prognostic impact remains uncertain in stable CAD. The key issue of a costeffective allocation for additional cost-intensive treatment options will be progress of a personalized individual risk stratification approach.

\section{Conclusions}

Secondary prevention in CAD holds strong potential to 


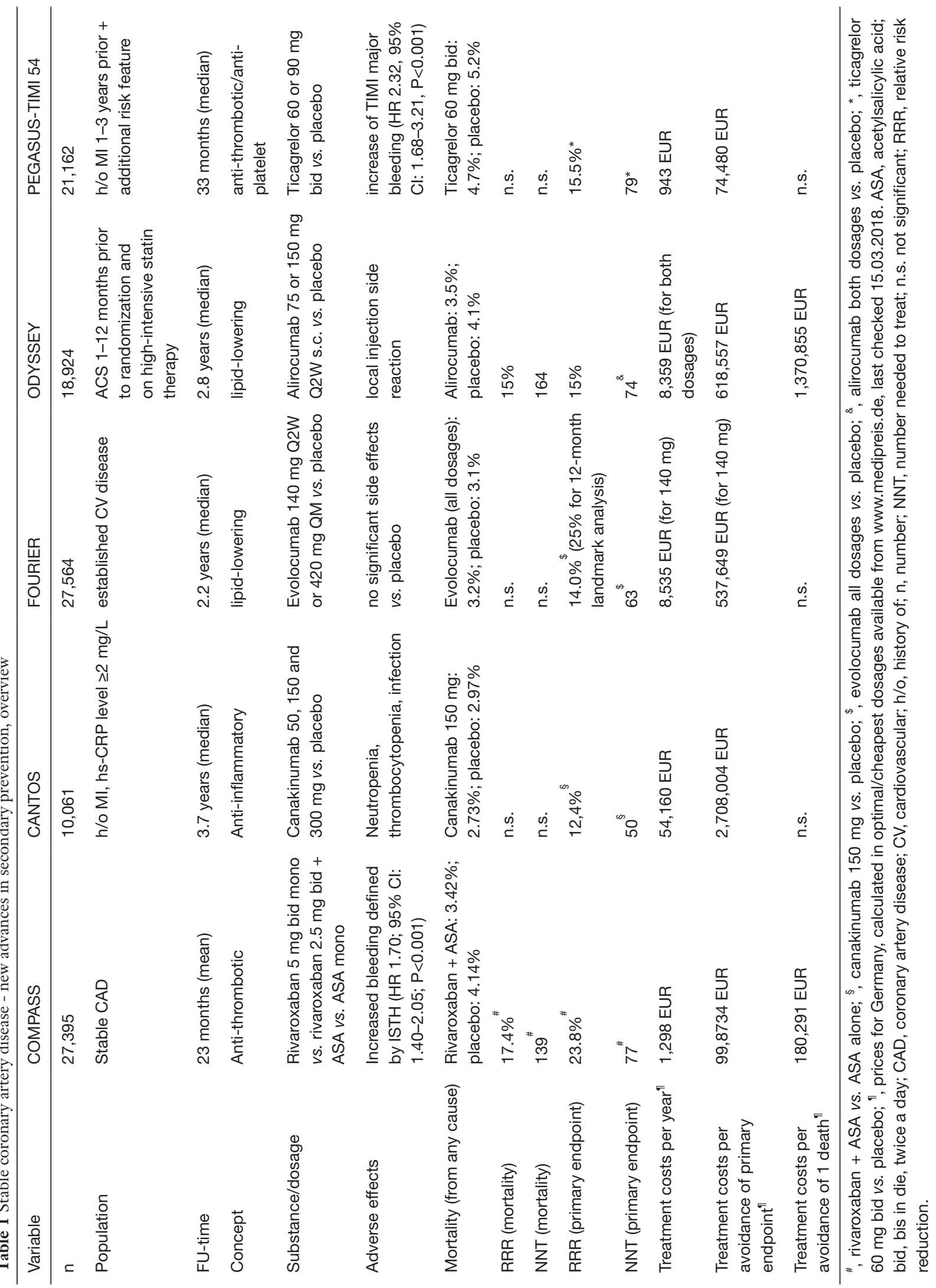


reduce $\mathrm{CV}$ events, even $\mathrm{CV}$ death. For patients with a high $\mathrm{CV}$ risk profile, it should therefore be one of the key goals to reduce the residual risk and consecutively subsequent events. Since so far little randomized data was available, it is great to see studies addressing this urgent need.

Achieving a target LDL level of $70 \mathrm{mg} / \mathrm{dL}$ seems obligatory, and PCSK9-inhibitors may play a certain role here after consequent utilization of conventional lipidlowering therapies. Whether further LDL reduction to levels around $50 \mathrm{mg} / \mathrm{dL}$ are the next step for each patient, needs to be discussed. While the anti-inflammatory CANTOS data is scientifically impressive, it needs not be seen whether the market will uptake a treatment strategy that is significantly more expensive (at least given the current prices). Probably, a one-shot treatment with continuation in those with reduced hs-CRP levels will be one way forward in patients with CKD.

At all, new options for secondary therapy are on the horizon or already available. Therefore, strategies to identify patients for each regime have to consider all, the individual efficacy, risk, and costs.

\section{Acknowledgements}

None.

\section{Footnote}

Conflicts of Interest: D Westermann has received travel expenses and lecture honoraria from Bayer AG, Novartis Pharma and from AstraZeneca. D Westermann is also in the in the advisory board of Bayer AG, Novartis Pharma and AstraZeneca. C Waldeyer has received lecture honoraria from AstraZeneca and travel expenses, lecture and consulting honoraria from Amgen. S Blankenberg has received honorary from Amgen und AstraZeneca. D Kalbacher has no conflicts of interest to declare.

\section{References}

1. Eckel RH, Jakicic JM, Ard JD, et al. 2013 AHA/ ACC Guideline on Lifestyle Management to Reduce Cardiovascular Risk. J Am Coll Cardiol 2014;63:2960-84.

2. Smith SC Jr, Benjamin EJ, Bonow RO, et al. AHA/ACCF secondary prevention and risk reduction therapy for patients with coronary and other atherosclerotic vascular disease: 2011 update: a guideline from the American Heart Association and American College of Cardiology
Foundation endorsed by the World Heart Federation and the Preventive Cardiovascular Nurses Association. J Am Coll Cardiol 2011;58:2432-46.

3. Perk J, De Backer G, Gohlke H, et al. European Guidelines on cardiovascular disease prevention in clinical practice (version 2012): The Fifth Joint Task Force of the European Society of Cardiology and Other Societies on Cardiovascular Disease Prevention in Clinical Practice. Eur Heart J 2012;33:1635-701.

4. Bestehorn K, Bauer T, Fleck E, et al. Coronary procedures in German hospitals: a detailed analysis for specific patient clusters. Clin Res Cardiol 2015;104:555-65.

5. Emond M, Mock MB, Davis KB, et al. Long-term survival of medically treated patients in the Coronary Artery Surgery Study (CASS) Registry. Circulation 1994;90:2645-57.

6. Pekkanen J, Linn S, Heiss G, et al. Ten-year mortality from cardiovascular disease in relation to cholesterol level among men with and without preexisting cardiovascular disease. N Engl J Med 1990;322:1700-7.

7. Sedlis SP, Jurkovitz CT, Hartigan PM, et al. Optimal Medical Therapy With or Without Percutaneous Coronary Intervention for Patients With Stable Coronary Artery Disease and Chronic Kidney Disease. Am J Cardiol 2009;104:1647-53.

8. Hansson GK. Inflammation, atherosclerosis, and coronary artery disease. N Engl J Med 2005;352:1685-95.

9. Mora S, Wenger NK, Demicco DA, et al. Determinants of residual risk in secondary prevention patients treated with high- versus low-dose statin therapy: the Treating to New Targets (TNT) study. Circulation 2012;125:1979-87.

10. Reiner $\check{Z}$, De Backer G, Fras Z, et al. Lipid lowering drug therapy in patients with coronary heart disease from 24 European countries-Findings from the EUROASPIRE IV survey. Atherosclerosis 2016;246:243-50.

11. Ridker PM. Residual inflammatory risk: addressing the obverse side of the atherosclerosis prevention coin. Eur Heart J 2016;37:1720-2.

12. Ridker PM, Cannon CP, Morrow D, et al. C-Reactive Protein Levels and Outcomes after Statin Therapy. N Engl J Med 2005;352:20-8.

13. Bohula EA, Giugliano RP, Cannon CP, et al. Achievement of dual low-density lipoprotein cholesterol and highsensitivity C-reactive protein targets more frequent with the addition of ezetimibe to simvastatin and associated with better outcomes in IMPROVE-IT. Circulation 2015;132:1224-33.

14. Fitó M, Guxens M, Corella D, et al. Effect of a Traditional 
Mediterranean Diet on Lipoprotein Oxidation. Arch Intern Med 2007;167:1195-203.

15. Laufs U, Karmann B, Pittrow D. Atorvastatin treatment and LDL cholesterol target attainment in patients at very high cardiovascular risk. Clin Res Cardiol 2016;105:783-90.

16. Cholesterol Treatment Trialists' (CTT) Collaboration, Fulcher J, O'Connell R, et al. Efficacy and safety of LDLlowering therapy among men and women: meta-analysis of individual data from 174000 participants in 27 randomised trials. Lancet 2015;385:1397-405.

17. Mega JL, Braunwald E, Wiviott SD, et al. Rivaroxaban in patients with a recent acute coronary syndrome. $\mathrm{N}$ Engl J Med 2012;366:9-19.

18. Bonaca MP, Bhatt DL, Cohen M, et al. Long-Term Use of Ticagrelor in Patients with Prior Myocardial Infarction. N Engl J Med 2015;372:1791-800.

19. Eikelboom JW, Connolly SJ, Bosch J, et al. Rivaroxaban with or without Aspirin in Stable Cardiovascular Disease. N Engl J Med 2017;377:1319-30.

20. Sabatine MS, Giugliano RP, Keech AC, et al. Evolocumab

Cite this article as: Kalbacher D, Waldeyer C, Blankenberg $\mathrm{S}$, Westermann D. Beyond conventional secondary prevention in coronary artery disease-what to choose in the era of CANTOS, COMPASS, FOURIER, ODYSSEY and PEGASUS-TIMI 54? A review on contemporary literature. Ann Transl Med 2018;6(16):323. doi: 10.21037/atm.2018.08.03 and Clinical Outcomes in Patients with Cardiovascular Disease. N Engl J Med 2017;376:1713-22.

21. Ridker PM, Everett BM, Thuren T, et al. Antiinflammatory Therapy with Canakinumab for Atherosclerotic Disease. N Engl J Med 2017; 377:1119-31.

22. Collins R, Reith C, Emberson J, et al. Interpretation of the evidence for the efficacy and safety of statin therapy. Lancet 2016;388:2532-61.

23. Sechtem U. Is FAME 2 a breakthrough for PCI in stable coronary disease? Clin Res Cardiol 2015;104:283-7.

24. Landmesser U, Chapman MJ, Farnier M, et al. European Society of Cardiology/European Atherosclerosis Society Task Force consensus statement on proprotein convertase subtilisin/kexin type 9 inhibitors: practical guidance for use in patients at very high cardiovascular risk. Eur Heart J 2017;38:2245-55.

25. Ridker PM, MacFadyen JG, Thuren T, et al. Effect of interleukin- $1 \beta$ inhibition with canakinumab on incident lung cancer in patients with atherosclerosis: exploratory results from a randomised, double-blind, placebocontrolled trial. Lancet 2017;390:1833-42. 